\title{
A novel method for screening of potential probiotics for high adhesion capability
}

\author{
Xin Wang, ${ }^{*} \dagger$ Qinglong Wu,‡ Kan Deng, ${ }^{*}$ Qiang Wei, ${ }^{*}$ Ping Hu, ${ }^{*}$ Jingjing He, ${ }^{*}$ Huan Liu, ${ }^{*}$ Yong Zheng, ${ }^{*}$ \\ Hua Wei,§ Nagendra P. Shah, $\ddagger^{1}$ and Tingtao Chen*1 \\ *Institute of Translational Medicine, and \\ †Institute of Life Science, Nanchang University, Nanchang, Jiangxi 330031, PR China \\ †Food and Nutritional Science, School of Biological Sciences, The University of Hong Kong, Pokfulam Road, Hong Kong \\ §State Key Laboratory of Food Science and Technology, Nanchang University, Jiangxi 330047, China
}

\begin{abstract}
To screen for potential probiotics with high adhesion capability, a chemostat model-based cultured human feces and denaturing gradient gel electrophoresis methods were applied, and the adhesion capability of the isolates was evaluated in vitro and in vivo. Lactobacillus plantarum (HM218749), Lactobacillus reuteri (EU547310), and Enterococcus faecalis (HM218543) were isolated from the slime on the chemostat wall, as these organisms could grow better at $37^{\circ} \mathrm{C}$ in an anaerobic environment and could resist harsh conditions (pH 1.5 and $0.30 \%$ bile salt). Lactobacillus plantarum, L. reuteri, and E. faecalis could adhere to HT-29 cells and reduce the adhesion of Shigella dysenteriae 2457, Staphylococcus aureus Cowan1, Enterobacter sakazakii 45401, and Escherichia coli 44102 to HT-29 cells. Moreover, the animal experiment showed that L. plantarum could adhere to mice intestine, increasing the number of lactobacilli and decreasing the number of enterococci.
\end{abstract}

Key words: probiotics, denaturing gradient gel electrophoresis, Lactobacillus plantarum, chemostat

\section{INTRODUCTION}

Increased awareness of health and busy lifestyles of consumers have created a strong and dynamic probiotics market (Granato et al., 2010). Probiotics, which are defined as "live microorganisms which when administered in adequate amounts, confer a health benefit on the host," are popular areas of study for microbiologists and nutritionists (Diplock et al., 1999; Hill et al., 2014). In addition to survival during gastrointestinal transit, the ability of probiotics to adhere to the intes-

\footnotetext{
Received January 19, 2015.

Accepted March 15, 2015.

${ }^{1}$ Corresponding authors: chentingtao1984@163.com and npshah@ hku.hk
}

tinal mucosa is one of the main selection criteria for probiotics (McNaught and MacFie, 2001; Ouwehand et al., 2002). Many health effects, including antagonism against pathogens, modulation of the immune system, and enhanced healing of damaged intestinal mucosa, are considered related to the adhesion of probiotics to intestinal mucosa, although strong adhesion of probiotics may only sometimes be associated with transient colonization (Johansson et al., 1993, Elliott et al., 1998).

Different probiotics exhibit different colonization capabilities and adhesion processes involve nonspecific adhesion (depending on the sticky substance, such as proteinaceous compounds, teichoic and lipoteichoic acids, peptidoglycans, and exopolysaccharides) and specific adhesion (involving interaction with the mucus layer of the gastrointestinal tract or with the proteins of the extracellular matrix through cell envelope and secreted proteins; Juntunen et al., 2001; Siciliano and Mazzeo, 2012). In our previous studies (Chen et al., 2011c, 2014a), we used chemostat models of the human large bowel, in which sterile medium enters and exits at a fixed rate and physicochemical variables (e.g., pH, temperature, and medium components) do not change, and a steady state or stable flora is usually achieved for 7 to $10 \mathrm{~d}$ after inoculation. In those experiments we found that some human fecal microflora could nonspecifically adhere to the chemostat wall and form a thick film, indicating their high adherence capability. Hence, a chemostat was designed to obtain a viscous membrane on a glass surface, and probiotic characteristics of the bacteria that were identified as having potential adhering ability were assessed both in vitro and in vivo.

\section{MATERIALS AND METHODS}

\section{Chemostat}

The chemostat was composed of units based on the same concept as that developed previously for the fecal 
or colonic system (Gibson et al., 1988; Chen et al., 2014a). The culture medium was (g/L): pectin (Sigma, St. Louis, MO), 0.6; xylan (Sigma), 0.6; arabinogalactan (Sigma), 0.6; amylopectin (Sigma), 0.6; Lintner starch (Sigma), 5.0; casein (Sigma), 3.0; peptone (Sigma), 3.0; $\mathrm{K}_{2} \mathrm{HPO}_{4}$ (Aoboxing, Beijing, China), 2.0; $\mathrm{NaHCO}_{3}$ (Aoboxing), 0.2; $\mathrm{NaCl}$ (Aoboxing), 4.5; $\mathrm{MgSO}_{4} \cdot 7 \mathrm{H}_{2} \mathrm{O}$ (Aoboxing), $0.5 ; \mathrm{CaCl}_{2} \cdot 2 \mathrm{H}_{2} \mathrm{O}$ (Aoboxing), 0.45 ; cysteine (Sigma), $0.4 ; \mathrm{FeSO}_{4} \cdot 7 \mathrm{H}_{2} \mathrm{O}$ (Aoboxing), 0.005; hemin (Sigma), 0.01; bile salts (Sigma), 0.05; Tween 80 (Aoboxing), $2 \mathrm{~mL}$; vitamin solution (Sigma), $1 \mathrm{~mL}$. One milliliter was pumped into the vessel. Culture fluid $(500 \mathrm{~mL})$ was stirred, sparged with gas mixture $\left(85 \% \mathrm{~N}_{2}, 5 \% \mathrm{H}_{2}, 10 \% \mathrm{CO}_{2}\right)$, and kept at $37^{\circ} \mathrm{C}$ and between $\mathrm{pH} 6.4$ and 6.6. Fresh medium was pumped into the culture vessel at $35 \mathrm{~mL} / \mathrm{h}$ (a dilution rate of $0.07 / \mathrm{h}$ ) and the excess medium was passed from the chemostat to a collecting vessel via a side arm (Carman et al., 2004).

Eight adult volunteers signed informed consent and donated their feces, which were stored at $-70^{\circ} \mathrm{C}$ (Chen et al., 2014a). Before inoculation into the chemostat, feces were thawed at room temperature for $1 \mathrm{~h}$ and uniformly suspended in prereduced and anaerobically sterilized diluent (culture medium) at a feces-to-diluent ratio of 1:4 and pooled together for the inoculation. The chemostat was inoculated with $50 \mathrm{~mL}$ of the suspension containing $10 \mathrm{~g}$ of feces on $\mathrm{d} 1,3$, and 5 by injecting through the septum of the lid of the vessel.

The chemostat was run for $31 \mathrm{~d}$ after inoculation to ensure a steady state was reached. From d 23 to 31, the thick film on the glass wall of the chemostat was aseptically collected and used for denaturing gradient gel electrophoresis (DGGE) analysis and cell culture experiments.

\section{Cultivable Cell Counts and Identification by Colony and Bacterial Morphology}

Bacterial counts were enumerated as previously reported (Harmsen et al., 2000). Diluted aliquots were replica-plated onto brain heart infusion (BHI; BHI; Difco Laboratories, Detroit, MI) agar (an enriched nonselective medium for the total bacterial count), de Man, Rogosa, Sharpe (MRS: Difco) agar (for Lactobacillus), Slanetz-Bartley medium agar (for Enterococcus), yeast potato dextrose (YPD; Difco) agar (for fungi), and MacConkey agar (for Escherichia coli; Difco). Plates were then incubated anaerobically in the anaerobic system (full of $85 \% \mathrm{~N}_{2}, 5 \% \mathrm{H}_{2}, 10 \% \mathrm{CO}_{2}$ ) at $37^{\circ} \mathrm{C}$ for 24 to $36 \mathrm{~h}$ followed by counting colonies from plates showing 25 to 250 colonies. The microbes were identified accord- ing to their colony and bacterial morphology, as well as based on their nutrients and oxygen requirements.

\section{DGGE Analysis}

The DNA was isolated by a bead-beating method (GC clamp sequence: CGCCCGGGGCGCGCCCCGGGCGGGGCGGGGGCACGGGGGG) and the bacterial primers (V3-R518, ATTACCGCGGCTGCTGG; V3-F357-GC, GC clamp: TACGGGAGGCAGCAG), Lactobacillus primers (Lac1, AGCAGTAGGGAA TCTTCCA; Lac2GC, GC clamp: ATTYCACCGCTACACATG), and fungal primers [FF390(F), CGATAACGAACGAGACCT; FR1 (R) GC clamp: AICCATT CAATCGGTAIT] were used for the DGGE analysis (Chen et al., 2011b).

The bands of interest in DGGE gels were excised using a sterile blade and incubated overnight at $4^{\circ} \mathrm{C}$ in Tris-EDTA buffer ( $\mathrm{pH}$ 8.0) to allow diffusion of DNA for further amplifications. The PCR products for sequencing were purified using the QIA quick PCR purification kit and subcloned with the pMD18-T vector system I (Takara, Dalian, China) according to the manufacturer's instructions. The transformants were randomly picked and sequenced by Invitrogen (Shanghai, China).

\section{Acid, Salt, Temperature, and Oxygen Tolerance of Isolates}

The isolates were grown in corresponding media at $37^{\circ} \mathrm{C}$ overnight and subcultured 3 times, then the cultures were centrifuged at $4,500 \times g$ for $10 \mathrm{~min}$ at $4^{\circ} \mathrm{C}$ to obtain the cell pellets of the isolates. For acid tolerance, each isolate was diluted 1:100 ( $\mathrm{vol} / \mathrm{vol}$ ) in PBS at $\mathrm{pH} 1.5,2.5,3.5,4.5$, and 7.0 and incubated for $4 \mathrm{~h}$. For bile salt tolerance, freshly prepared cultures were inoculated into corresponding media containing 0.1 to $0.3 \%$ (wt/wt) bile salts and incubated at $37^{\circ} \mathrm{C}$ for a further $4 \mathrm{~h}$. Growth at different temperatures $(25,30$, 37,42 , and $46^{\circ} \mathrm{C}$ ) and that under anaerobic or aerobic conditions were also evaluated. All the operations were carried out in the anaerobic system and all bacterial colonies were enumerated using the plate count method (Chen et al., 2014b).

\section{HT-29 Cell Cultures and Adherence Assay}

The HT-29 cells were stored in a liquid nitrogen tank with $90 \%$ fetal bovine serum (Hyclone Laboratories, Ogden, UT) and 10\% dimethyl sulfoxide. The cells were cultured in complete RPMI-1640 medium (Hyclone 
Laboratories) supplemented with $10 \%$ (vol/vol) fetal bovine serum in an incubator (HEPA class 100, Thermo Scientific, Waltham, MA) with $5 \% \mathrm{CO}_{2}$ and $95 \%$ air at $37^{\circ} \mathrm{C}$. The culture medium was changed daily until HT29 monolayers were at 80 to $90 \%$ confluence.

Adherence of probiotics to HT-29 cells was examined as described previously (Zhang et al., 2010). The HT29 monolayers, which were prepared on glass coverslips and placed in 6-well plates (Corning Inc., Corning, $\mathrm{NY})$, were washed twice with PBS ( $\mathrm{pH} 7.4)$. A 1-mL aliquot of probiotics $(8 \log \mathrm{cfu} / \mathrm{mL}$ ) and $1 \mathrm{~mL}$ of the cell line culture medium were added to each well of the tissue culture plate and the plate was incubated at $37^{\circ} \mathrm{C}$ in $5 \% \mathrm{CO}_{2}$. After $2 \mathrm{~h}$ of incubation, monolayers were washed 4 times with sterile PBS, fixed with methanol for $30 \mathrm{~min}$, Gram stained, and examined microscopically. An adherence index was determined from 20 random microscopic fields of adhering probiotics per 100 HT-29 cells. Each adherence assay was carried out in triplicate.

\section{Inhibition of Adhesion of Foodborne Pathogens to HT-29 Cells}

The ability of probiotics to inhibit adhesion of foodborne pathogens to HT-29 cells was assayed following the method reported previously (Zhang et al., 2010). The Luria-Bertani medium (for laboratory strains Shigella dysenteriae 2457, Staphylococcus aureus Cowan1, Enterobacter sakazakii 45401, and Escherichia coli 44102) and YPD (for laboratory strain Candida albicans SC5314) medium were used to culture the pathogens at $37^{\circ} \mathrm{C}$ overnight and subcultured for 3 times; Approximately $3 \times 10^{5}$ HT-29 cells per well were seeded in a 12-well plate (Corning Inc.), culture medium was changed daily, and HT-29 monolayers at 80 to $90 \%$ confluence were washed twice with PBS (pH 7.4) before the experiments. In the exclusion assay, HT-29 monolayers were inoculated with $300 \mathrm{~mL}$ of the suspension containing probiotics ( $7 \mathrm{log} \mathrm{cfu} /$ well) in RPMI-1640 medium and incubated for $1 \mathrm{~h}$ at $37^{\circ} \mathrm{C}$ in $5 \% \mathrm{CO}_{2}$. The HT-29 monolayers were then inoculated with $100 \mathrm{~mL}$ of foodborne pathogens (7 log cfu/well) in RPMI-1640 medium and incubated for another $1 \mathrm{~h}$.

In all experiments, nonadhering cells were removed by washing 4 times with sterile PBS and then treated with $0.5 \mathrm{~mL}$ of $0.5 \%$ (vol/vol) Triton X-100 (Amresco, Solon, $\mathrm{OH}$ ) for $5 \mathrm{~min}$ in an ice water bath. The cells were then serially diluted and plated onto LB plates for counting foodborne pathogens. The ability of probiotics to exclude foodborne pathogens was determined by comparing adhesion of foodborne pathogens in the presence of probiotics to that of foodborne pathogens.
The number of foodborne pathogen cells prevented from adhering to HT-29 cells was then expressed as a percentage value. All experiments were independently carried out 3 times and each assay was performed in triplicate.

\section{Animal Model and Experimental Design}

Six- to 8-wk-old specific-pathogen-free BALB/c female mice $(18 \pm 2 \mathrm{~g})$ were purchased from the Hebei Municipal Center for Disease Control and Prevention (Hebei, China). After 1 wk of adjustment in the Laboratory Animal Center of Nanchang University, the feces of mice $(n=8)$ were collected before experiments (control stage); then probiotics were fed for $2 \mathrm{wk}$ (treatment stage), followed by collection of feces 2 wk after stopping feeding of the probiotics (recovery stage). An aliquot of the feces was used for microbial count and the rest of the samples were stored at $-20^{\circ} \mathrm{C}$ for the DGGE analysis.

\section{Statistical Analysis}

Data of viable cell counts were submitted to ANOVA, and then significant differences between means were determined by the nonparametric Student-NewmanKeuls separation test with significance level $(P<0.05)$ or very significant level $(P<0.01)$.

\section{RESULTS AND DISCUSSION}

\section{Isolation of Bacteria from the Chemostat Wall Using Viable Cell Count and DGGE}

To isolate bacteria with strong adherence ability to the human intestine, human feces were obtained from human volunteers and cultured in chemostat for 31 $\mathrm{d}$ to allow the formation of a microbial layer on the chemostat wall. Then the bacterial layer was aseptically collected and smeared on to BHI, MRS, and YPD plates and incubated at 25 or $37^{\circ} \mathrm{C}$ under anaerobic conditions. Forty-one different colonies (16 from BHI, 16 from MRS, and 9 from YPD) were picked based on their cell and colony morphologies and their identity was determined using DGGE method (Chen et al., 2011a). In principle, the same bacteria exhibited the same band number and band location in DGGE gel; hence, the microbes corresponding to lanes 5, 7, and 14 as shown in Figure 1A, lanes 5, 11, and 14 as shown in Figure 1B, and lanes 5 and 7 as shown in Figure $1 \mathrm{C}$ were sent for sequencing (Shenggong Biotech, Shanghai, China). Three different bacteria, namely Lactobacillus plantarum (HM218749, on BHI and MRS 

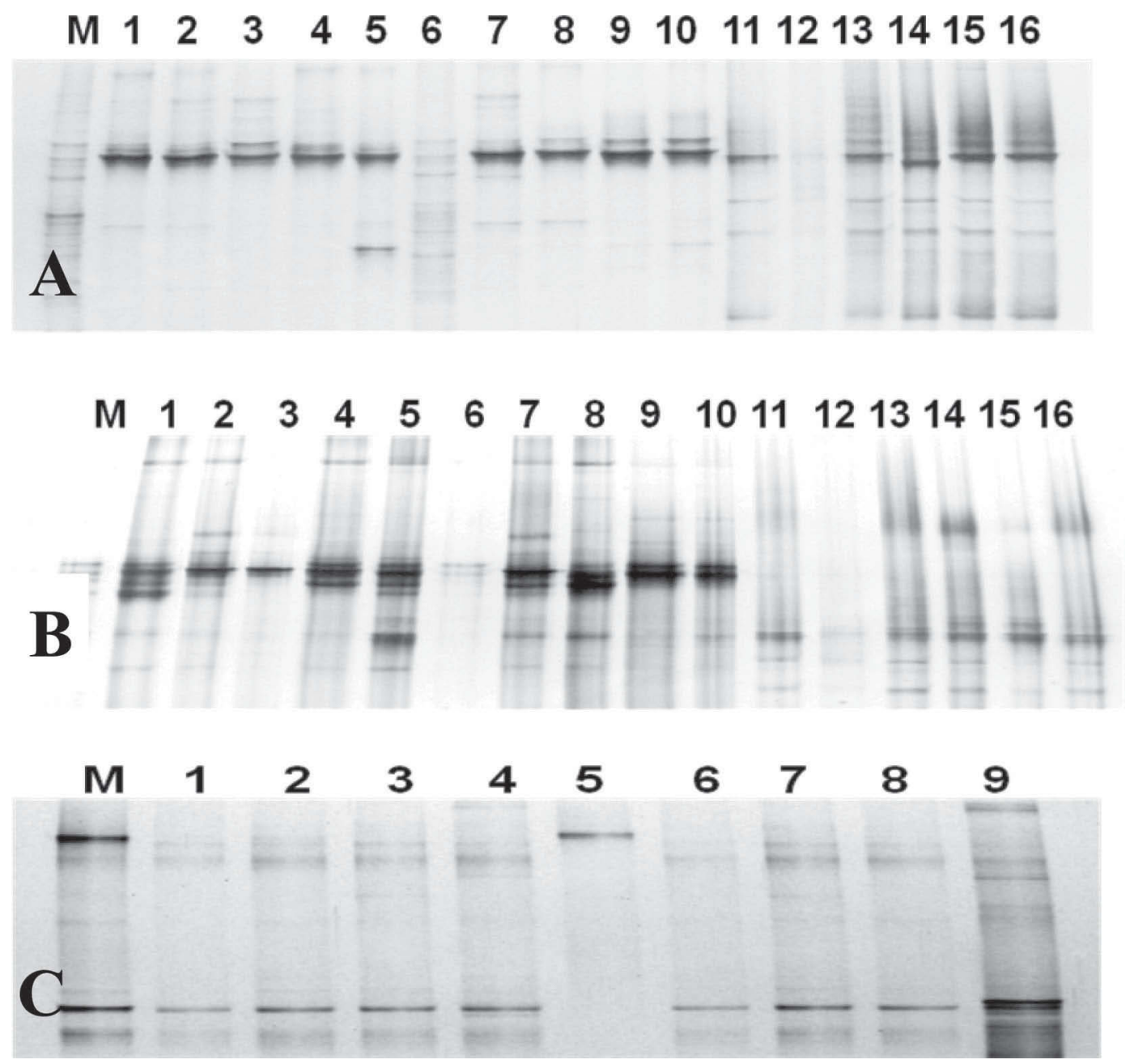

Figure 1. Classification of bacteria using specific primers in denaturing gradient gel electrophoresis (DGGE) gel. (A) Bacterial DGGE gel; M $=$ mixture of all the bacteria; $1-10=$ strains isolated from de Man, Rogosa, Sharpe (MRS) medium; 11-16 = strains isolated from brain heart infusion medium. (B) Bacillus DGGE gel; $\mathrm{M}=$ mixture of all the bacteria; $1-10=$ strains isolated from MRS medium; $11-16=$ strains isolated from BHI medium. (C) Fungal DGGE gel; $\mathrm{M}=$ mixture of all fungi; 1-9 = strains isolated from yeast potato dextrose medium.

plates), Lactobacillus reuteri (EU547310, on BHI and MRS plates), and Enterococcus faecalis (HM218543, on BHI and YPD plates), were also identified (Table 1).

\section{Evaluation of Acid, Salt, Temperature, and Oxygen Tolerance of Isolates}

It is crucial for probiotics to be viable to exert their beneficial effects; hence, tolerance to acid and bile salt has become an important selection criterion for probiotic organisms (Jensen et al., 2012). It is anticipated that foods will stay in the stomach for 2 to $3 \mathrm{~h}$ at low $\mathrm{pH}$ (about 3.0) and in the intestine at high salt concentrations (about 0.3\%). As shown in Figure 2, the isolated L. plantarum, L. reuteri, and E. faecalis could tolerate a low $\mathrm{pH}$ of 1.5 and exhibited high biomass at 7 to $8 \log \mathrm{cfu} / \mathrm{mL}$ for up to $4 \mathrm{~h}$. The tolerance of isolates to bile salt was inferior compared with that of

Table 1. Identification of the microorganisms isolated from the chemostat

\begin{tabular}{lllll}
\hline Strain no. & Closest relative & Similarity (\%) & GenBank No. & $\begin{array}{l}\text { Sequence } \\
\text { length }^{1}(\mathrm{bp})\end{array}$ \\
\hline 1 & Lactobacillus plantarum & 99 & HM218749.1 & 1,450 \\
2 & Lactobacillus reuteri & 98 & EU547310.1 & 1,450 \\
3 & Enterococcus faecalis & 99 & HM218543.1 & 1,450 \\
\hline${ }^{1}$ Sequenced length of 16S rDNA V3 region. & & &
\end{tabular}



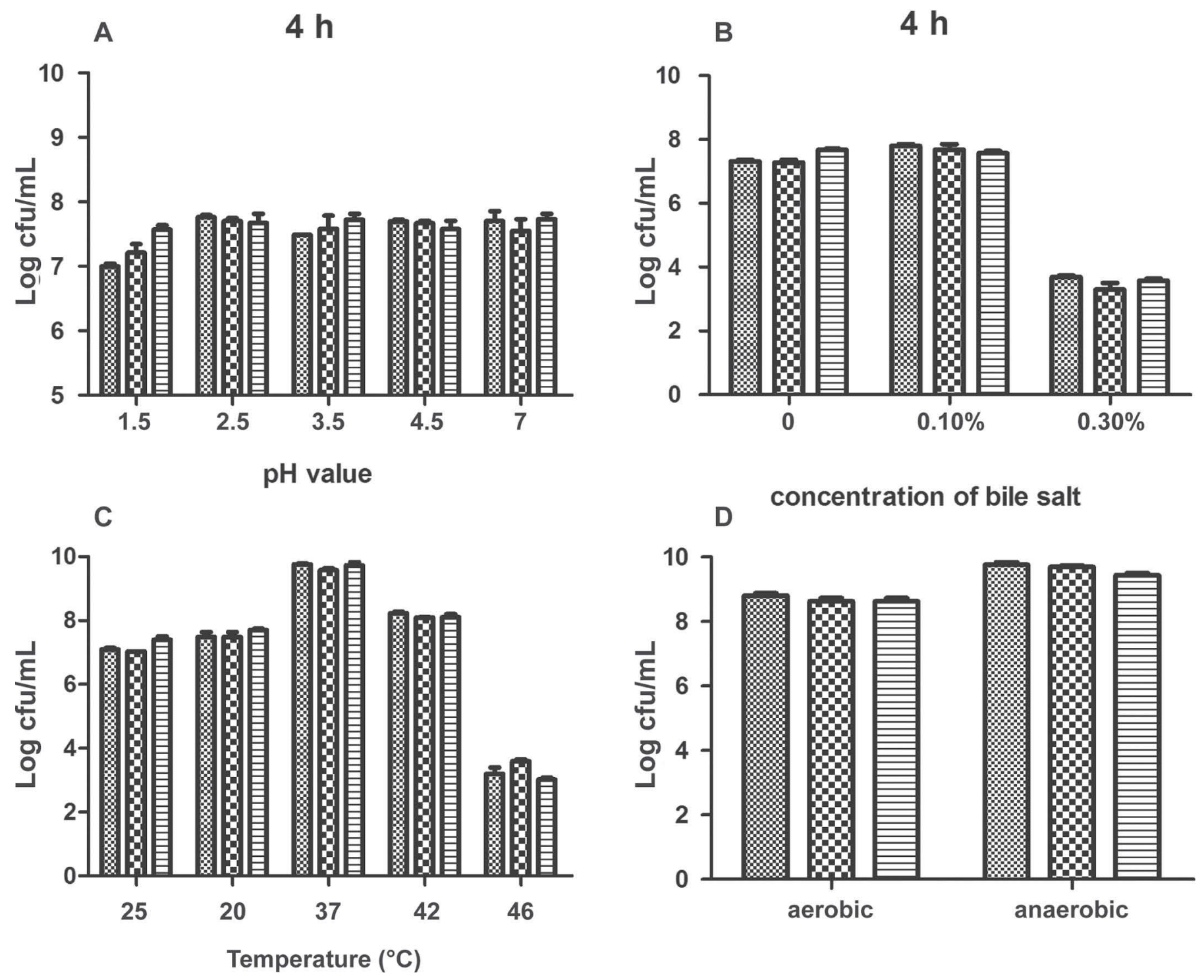

Figure 2. The probiotic characteristics of Lactobacillus plantarum, Lactobacillus reuteri, and Enterococcus faecalis. (A) $\mathrm{pH}$ tolerance; (B) bile salt tolerance; (C) temperature tolerance; (D) oxygen tolerance. Error bars represent SE.

$\mathrm{pH}$ tolerance, and their biomass at $0.3 \%$ bile salt was at 3 to $4 \log \mathrm{cfu} / \mathrm{mL}$. Additionally, a temperature of $37^{\circ} \mathrm{C}$ and anaerobic conditions were suitable for all isolates.

\section{Evaluation of Isolates for Ability to Adhere to Intestinal Cells and Exclude Pathogens}

Adhesion to mammalian epithelial cells is a key process for bacteria to colonize the gastrointestinal tract. Adhesion of pathogens allows release of enzymes and toxins initiating necrotic processes against target cells, thereby facilitating invasion (Jankowska et al., 2008), and probiotics could competitively bind to adhesion sites and produce components with antimicrobial activity to exclude pathogens from the gastrointestinal tract (Baccigalupi et al., 2005; Collado et al., 2006).

From Figure 3A, all the isolates exhibited strong adherence ability to HT-29 cells; L. plantarum possessed the highest adherence at 1,100 cfu/100 cells. With regard to antiadherence property to pathogens, $L$. 
A

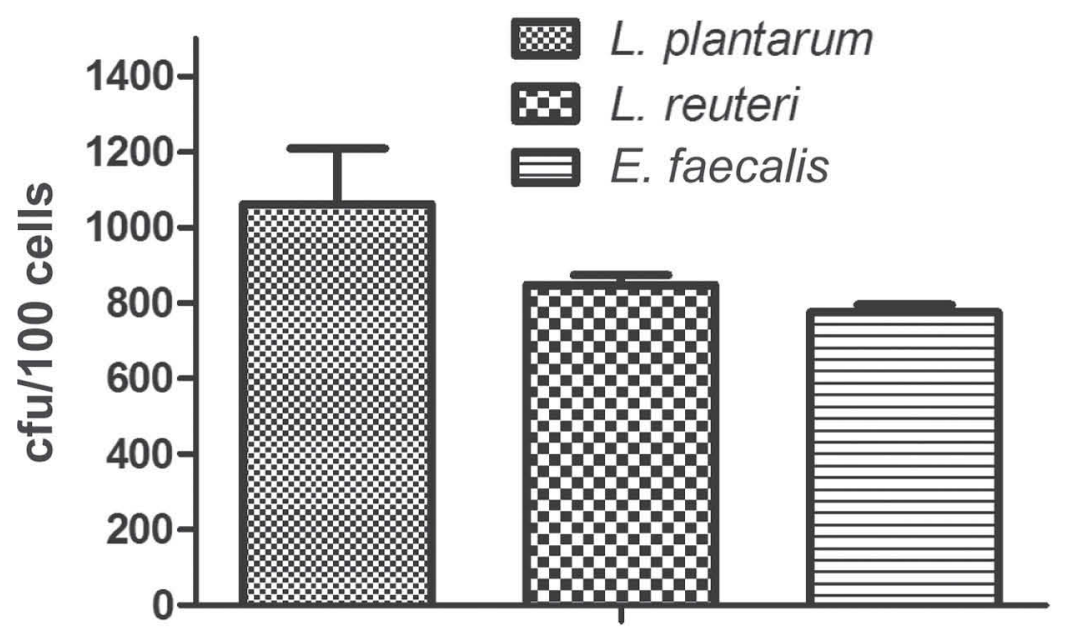

B
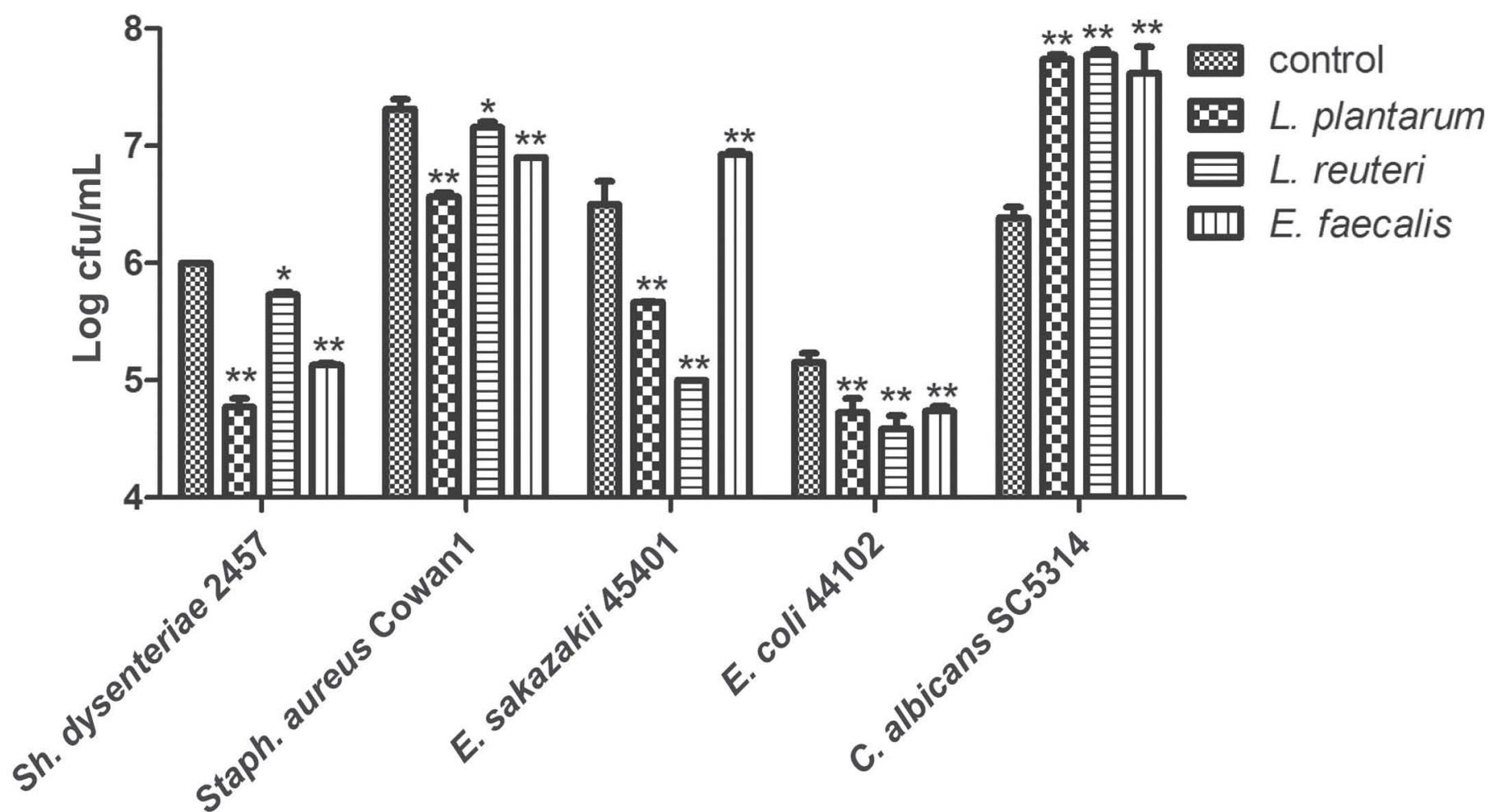

Figure 3. Adherence capability of the Lactobacillus plantarum, Lactobacillus reuteri, and Enterococcus faecalis on HT-29 cell line (A) and the antiadherence property of the isolates against pathogens (Shigella dysenteriae, Staphylococcus aureus Cowan 1, Enterobacter sakazakii, Escherichia coli, and Candida albicans) on HT-29 cell line (B). Error bars represent SE.

plantarum could also significantly lower the adherence of $S$. dysenteriae 2457, Staphylococcus aureus Cowan1, E. sakazakii 45401, and E. coli $44102(P<0.01)$. It is important to note that all the isolates enhanced, rather than lowered, the growth of fungal pathogen $C$. albicans SC5314. It may be that C. albicans SC5314 possessed different binding sites on to HT-29 cells compared with $L$. plantarum, thus $L$. plantarum exhib- 
A
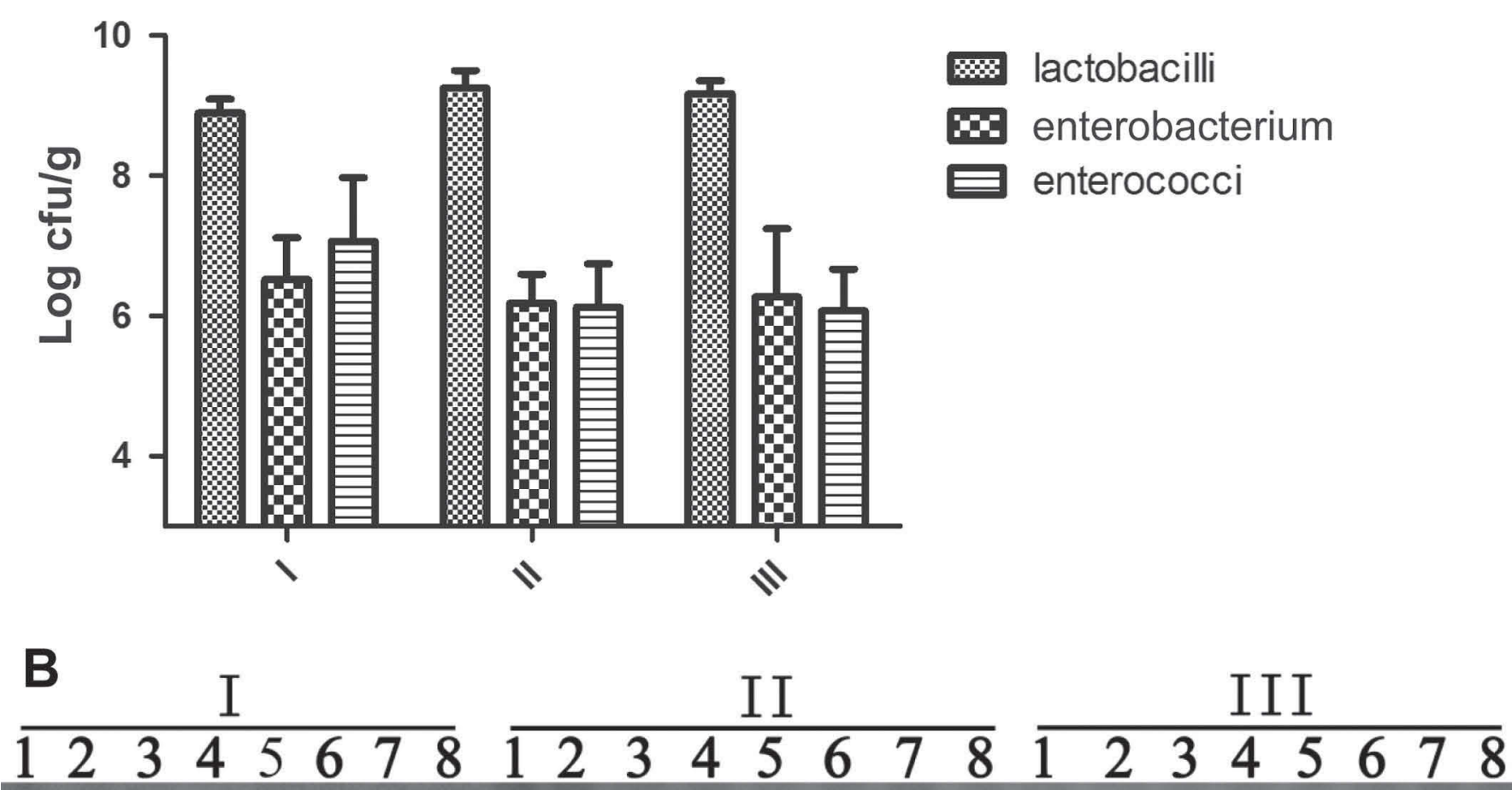

Figure 4. The microbial diversity in mice intestine. (A) The effect of Lactobacillus plantarum on the microbial number of lactobacilli, enterobacteria, and enterococci in control stage (I; before probiotics feeding); treatment stage (II; probiotics feeding for 2 wk); and recovery stage (III; stop probiotics feeding for 2 wk); error bars represent SE. (B) The effect of L. plantarum on microbial species in control stage (I; before probiotics feeding); treatment stage (II; probiotics feeding for $2 \mathrm{wk}$ ); and recovery stage (III; stop probiotics feeding for 2 wk). Bands in the white boxed area belong to L. plantarum and represent their existence in mice intestinal tract.

ited little antiadherence property against C. albicans SC5314.

\section{Evaluation of the Adherence of Isolates in Mice Intestines}

The human intestinal tract is a nutrient-rich environment packed with up to 100 trillion microbes (Whitman et al., 1998), and the microbial community has been considered as a factor that could influence predisposition to specific diseases in the context of certain hosts (Ley et al., 2006). In our previous studies, L. plantarum could strongly adhere to HT-29 cells (Figure 3), glass, stone, wood, and plastic; thus, L. plantarum was chosen for evaluating its adherence ability using a mice model. Before feeding L. plantarum to mice, it appeared that 3 
of 8 mice (mice 1, 3, and 5) were positive for this strain (Figure 4); however, during the treatment stage, the L. plantarum not only occupied the dominant position among Lactobacillus species in the intestine of the mice but also enhanced the microbial diversity, which could benefit host health. Two weeks after feeding without $L$. plantarum, this strain still exhibited dominance in the intestine of 6 of 8 mice, showing much more abundance than the control (Figure 4). In addition, the viable cell count results indicated that the $L$. plantarum could increase the number of Lactobacillus species and lower the number of Enterococcus species, suggesting a positive effect on host health (Cross, 2002).

\section{CONCLUSIONS}

In the present study, we developed a chemostat model to screen organisms for strong adherence ability, and $L$. plantarum was isolated using this model and its probiotic characteristics were determined. The organism showed tolerance to low $\mathrm{pH}$ and high concentrations of salt bile and possessed high adherence capability and antiadherence property against foodborne pathogens both in vivo and in vitro.

\section{ACKNOWLEDGMENTS}

The authors thank Hongbo Xin (Institute of Translational Medicine, Nanchang University) for his valuable methodologies. This work was supported by grants from Jiangxi Province (China; 20142BAB215066, GJJ14197, 2014A065), the Ministry of Science and Technology "Creation of major new drugs" special (2011ZX09102009-01), and the landing plan project of Jiangxi Province (China, QT43-3).

\section{REFERENCES}

Baccigalupi, L., A. Di Donato, M. Parlato, D. Luongo, V. Carbone, M. Rossi, E. Ricca, and M. De Felice. 2005. Small surface-associated factors mediate adhesion of a food-isolated strain of Lactobacillus fermentum to Caco-2 cells. Res. Microbiol. 156:830-836.

Carman, R. J., M. A. Simon, H. Fernández, M. A. Miller, and M. J. Bartholomew. 2004. Ciprofloxacin at low levels disrupts colonization resistance of human fecal microflora growing in chemostats. Regul. Toxicol. Pharmacol. 40:319-326.

Chen, T., S. Li, and H. Wei. 2014a. Antibiotic resistance capability of cultured human colonic microbiota growing in a chemostat model. Appl. Biochem. Biotechnol. 173:765-774.

Chen, T., M. Wang, S. Jiang, S. Xiong, and H. Wei. 2011a. The application of polymerase chain reaction-denaturing gradient gel electrophoresis (PCR-DGGE) method in microbial screening. Afr. J. Biotechnol. 10:9387-9395.

Chen, T., M. Wang, S. Jiang, S. Xiong, D. Zhu, and H. Wei. 2011b. Investigation of the microbial changes during koji-making process of Douchi by culture-dependent techniques and PCR-DGGE. Int. J. Food Sci. Technol. 46:1878-1883.

Chen, T., Q. Wu, S. Li, S. Xiong, S. Jiang, Q. Tan, Z. Zhang, D. Zhu, and H. Wei. 2014b. Microbiological quality and characteristics of probiotic products in China. J. Sci. Food Agric. 94:131-138.
Chen, T., J. Yuan, X. Feng, H. Wei, and W. Hua. 2011c. Effects of enrofloxacin on the human intestinal microbiota in vitro. Int. J. Antimicrob. Agents 37:567-571.

Collado, M. C., L. Jalonen, J. Meriluoto, and S. Salminen. 2006. Protection mechanism of probiotic combination against human pathogens: In vitro adhesion to human intestinal mucus. Asia Pac. J. Clin. Nutr. 15:570-575.

Cross, M. L. 2002. Microbes versus microbes: Immune signals generated by probiotic lactobacilli and their role in protection against microbial pathogens. FEMS Immunol. Med. Microbiol. 34:245-253.

Diplock, A. T., P. J. Aggett, M. Ashwell, F. Bornet, E. B. Fern, and M. B. Roberfroid. 1999. Scientific concepts in functional foods in Europe: Consensus document. Br. J. Nutr. 81:S1-S27.

Elliott, S. N., A. Buret, W. McKnight, M. J. Miller, and J. L. Wallace. 1998. Bacteria rapidly colonize and modulate healing of gastric ulcers in rats. Am. J. Physiol. 275:G425-G432.

Gibson, G. R., J. H. Cummings, and G. T. Macfarlane. 1988. Use of a three-stage continuous culture system to study the effect of mucin on dissimilatory sulfate reduction and methanogenesis by mixed populations of human gut bacteria. Appl. Environ. Microbiol. 54:2750-2755.

Granato, D., G. F. Branco, F. Nazzaro, A. G. Cruz, and J. A. Faria 2010. Functional foods and nondairy probiotic food development: Trends, concepts, and products. Compr. Rev. Food. Sci. Food Saf. 9:292-302.

Harmsen, H. J., G. Gibson, P. Elfferich, G. Raangs, A. Wildeboer-Veloo, A. Argaiz, M. Roberfroid, and G. Welling. 2000. Comparison of viable cell counts and fluorescence in situ hybridization using specific rRNA-based probes for the quantification of human fecal bacteria. FEMS Microbiol. Lett. 183:125-129.

Hill, C., F. Guarner, G. Reid, G. R. Gibson, D. J. Merenstein, B. Pot, L. Morelli, R. B. Canani, H. J. Flint, and S. Salminen. 2014. Expert consensus document: The International Scientific Association for Probiotics and Prebiotics consensus statement on the scope and appropriate use of the term probiotic. Nat. Rev. Gastroenterol. Hepatol. 11:506-514.

Jankowska, A., D. Laubitz, H. Antushevich, R. Zabielski, and E. Grzesiuk. 2008. Competition of Lactobacillus paracasei with Salmonella enterica for adhesion to Caco-2 cells. J. Biomed. Biotechnol. 2008:357964.

Jensen, H., S. Grimmer, K. Naterstad, and L. Axelsson. 2012. In vitro testing of commercial and potential probiotic lactic acid bacteria. Int. J. Food Microbiol. 153:216-222.

Johansson, M. L., G. Molin, B. Jeppsson, S. Nobaek, S. Ahrne, and S. Bengmark. 1993. Administration of different Lactobacillus strains in fermented oatmeal soup: In vivo colonization of human intestinal mucosa and effect on the indigenous flora. Appl. Environ. Microbiol. 59:15-20.

Juntunen, M., P. Kirjavainen, A. Ouwehand, S. Salminen, and E. Isolauri. 2001. Adherence of probiotic bacteria to human intestinal mucus in healthy infants and during rotavirus infection. Clin. Diagn. Lab. Immunol. 8:293-296.

Ley, R. E., D. A. Peterson, and J. I. Gordon. 2006. Ecological and evolutionary forces shaping microbial diversity in the human intestine. Cell 124:837-848.

McNaught, C., and J. MacFie. 2001. Probiotics in clinical practice: A critical review of the evidence. Nutr. Res. 21:343-353.

Ouwehand, A. C., T. Suomalainen, S. Tölkkö, and S. Salminen. 2002. In vitro adhesion of propionic acid bacteria to human intestinal mucus. Lait 82:123-130.

Siciliano, R. A., and M. F. Mazzeo. 2012. Molecular mechanisms of probiotic action: A proteomic perspective. Curr. Opin. Microbiol. 15:390-396.

Whitman, W. B., D. C. Coleman, and W. J. Wiebe. 1998. Prokaryotes: The unseen majority. Proc. Natl. Acad. Sci. USA 95:6578-6583.

Zhang, Y.-C., L.-W. Zhang, Y.-F. Tuo, C.-F. Guo, H.-X. Yi, J.-Y. Li, X. Han, and M. Du. 2010. Inhibition of Shigella sonnei adherence to HT-29 cells by lactobacilli from Chinese fermented food and preliminary characterization of S-layer protein involvement. Res. Microbiol. 161:667-672. 